\title{
CMEARTICLE
}

\section{Magnetic resonance imaging of diabetic foot complications}

Keynes TA $\underline{L O W}^{1}$, MMed, FRCR, Wilfred CG $\underline{P e h}^{1}$, FRCP, FRCR

\begin{abstract}
This pictorial review aims to illustrate the various manifestations of the diabetic foot on magnetic resonance (MR) imaging. The utility of MR imaging and its imaging features in the diagnosis of pedal osteomyelitis are illustrated. There is often difficulty encountered in distinguishing osteomyelitis from neuroarthropathy, both clinically and on imaging. By providing an accurate diagnosis based on imaging, the radiologist plays a significant role in the management of patients with complications of diabetic foot.
\end{abstract}

Keywords: diabetic foot, diabetes mellitus complications, foot infection, foot neuroarthropathy, osteomyelitis

\section{INTRODUCTION}

Diabetes mellitus (DM) is a growing health problem worldwide. The global prevalence of DM was approximately $5.1 \%$ in 2012 and is projected to hit $7.7 \%$ by $2030 .{ }^{(1)}$ This is contributed to by the increasingly sedentary lifestyles and evolving dietary trends associated with growing affluence, especially in developed nations. Similar to its geographical extent, DM afflicts the body in a global fashion, resulting in a myriad of complications involving multiple end-organs. In this article, we will consider diabetic foot complications, a significant cause of morbidity and mortality in diabetic patients. Diabetic foot complications comprise pathologies affecting osseous, joint, muscular, tendinous and other soft tissue structures. Infection of the soft tissue and bones is particularly common in the diabetic foot, and early diagnosis is crucial, as it permits timely treatment (e.g. antibiotics, surgical debridement). With delayed treatment and a background of impaired healing, deterioration toward a limb- and life-threatening stage is inevitable.

Almost all diabetic foot infections are due to direct spread from a skin ulcer. ${ }^{(2)}$ The diabetic foot is prone to skin ulceration due to associated risk factors such as microangiopathy with peripheral neuropathy, and altered biomechanics. Unrecognised trauma, in the background of these risk factors, results in superimposed infection and restricts healing. ${ }^{(3,4)}$ Another significant route of infection is related to surgery or penetrating trauma, where infectious material may be directly implanted. Haematogenous spread of infection rarely involves the foot but may be seen in the paediatric population, or with atypical organisms such as Mycobacterium tuberculosis. ${ }^{(5,6)}$ However, in these cases, the clinical diagnosis is straightforward.

Chronic repeated unperceived traumatic injuries to the joints of the foot results in neuroarthropathy (Charcot neuroosteoarthropathy). The diabetic patient is predisposed to this condition because of associated peripheral neuropathy and peripheral vascular disease. There is progressive arthropathy characterised by cartilage damage, bone erosions, subchondral cysts, joint deformities and new bone formation. This usually affects the tarsometatarsal joints, causing collapse of the longitudinal arch and a 'rocker-bottom' deformity that increases load-bearing on the cuboid. Both the acute and chronic forms of neuroarthropathy are recognised.

Neuroarthropathy and osteomyelitis often occur concurrently in the diabetic foot, but either of them may be more prominent. It is clinically and radiologically challenging to distinguish between these two entities. Both conditions may show marrow oedema and enhancement, joint effusion, as well as adjacent soft tissue oedema. Apart from analysing the magnetic resonance (MR) imaging features, correlation with clinical findings is also necessary in order to arrive at an accurate diagnosis. ${ }^{(7,8)}$ In this pictorial review, we illustrate the MR imaging appearances of diabetic foot complications, including osteomyelitis, and also discuss the MR imaging appearance of neuroarthropathy, both with and without coexisting infection.

\section{IMAGING MODALITIES}

Radiography is the preferred initial imaging modality, since it is easily available, inexpensive, and provides superb resolution of bones. However, in the case of early osteomyelitis, the rate and accuracy of detection is at best $50 \%-60 \%$, as the soft tissues are not adequately demonstrated. ${ }^{(9)}$ For the assessment of soft tissue infection and osteomyelitis involving the foot, MR imaging is the modality of choice, with sensitivity and specificity of $90 \%$ and $83 \%$, respectively. ${ }^{(10,11)}$ In addition, it allows preoperative mapping of the area of infection, potentially limiting the extent of resection. ${ }^{(12)}$ It has been shown that MR imaging, in combination with radiography, is the most accurate in the detection of diabetic pedal osteomyelitis (and its differentiation from neuroarthropathy). ${ }^{(13-17)}$

Bone scintigraphy and white cell scintiscans are able to demonstrate increased uptake in osteomyelitis, but they are of limited use due to their poor specificity. Ultrasonography is valuable as a widely available noninvasive imaging modality in the evaluation of soft tissue pathologies and localisation of foreign bodies. However, it has a limited role in the assessment of diabetic foot complications (particularly of the bones). ${ }^{(18)}$ 

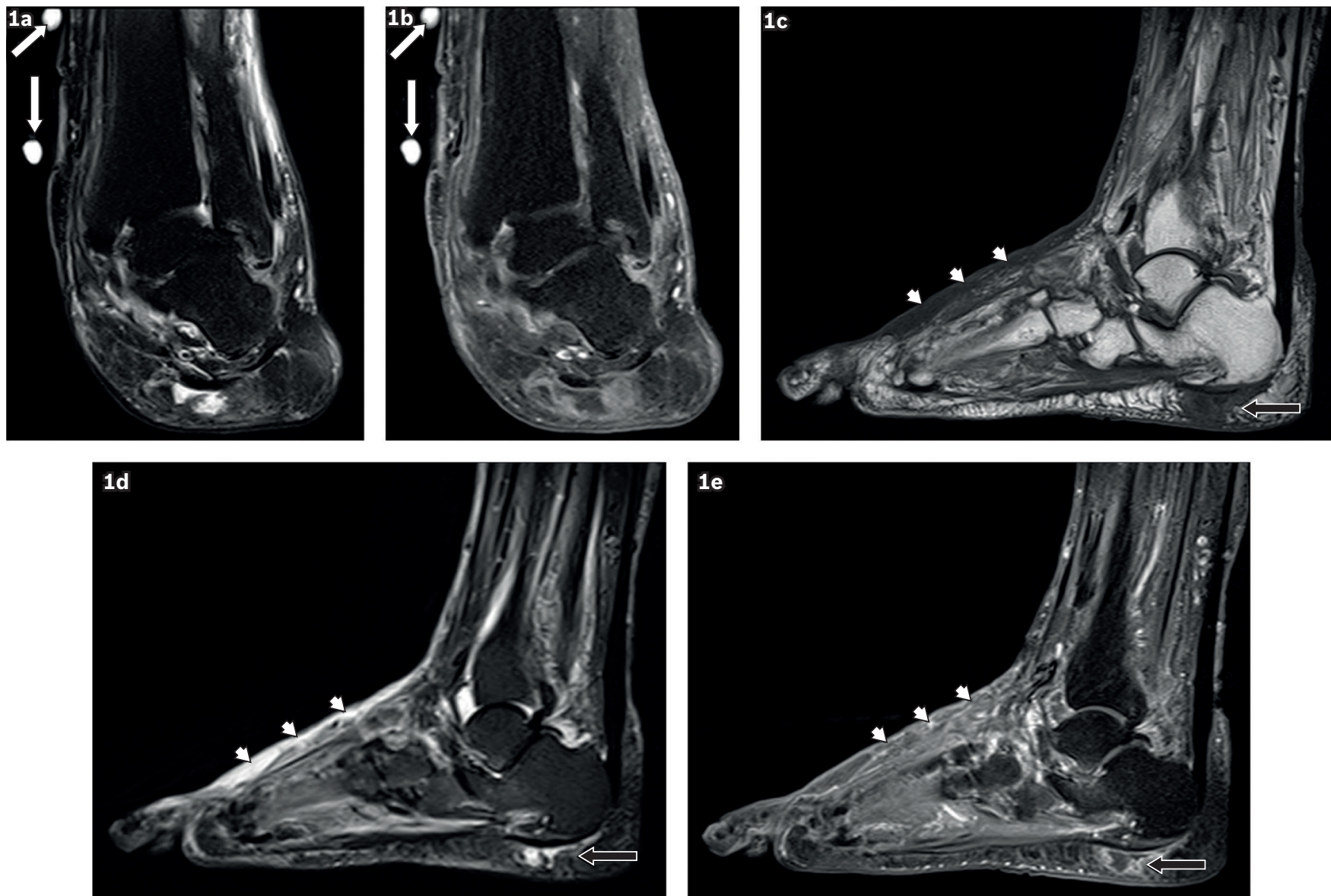

Fig. 1 A 59-year-old woman presented with a left ankle ulcer with signs of infection. Coronal (a) T2-W fat-suppressed (FS) and (b) contrast-enhanced T1-W FS MR images of the left ankle show external markers (white arrows), indicating the site of an ulcer along the medial aspect of the distal tibia, evident as a soft tissue defect with focal interruption of the cutaneous line and elevated margins from pre-existing callus formation. High T2-W signal and contrast enhancement at the base of this ulcer are due to granulation tissue. Sagittal (c) T1-W, (d) T2-W FS and (e) contrast-enhanced T1-W FS MR images of the left foot show a focal fluid collection with high T2-W and low T1-W signals in the subcutaneous plane of the heel at the insertion of the Achilles tendon, typical of an adventitial bursa (black arrows). In this case, peripheral enhancement suggests superimposed inflammation/infection. Surrounding cellulitis is present at the dorsum of the foot, seen as areas of T1-hypointense and T2-hyperintense signals within the subcutaneous tissues with enhancement of subcutaneous fat (arrowheads)

\section{MR IMAGING PROTOCOL}

The MR imaging should ideally be customised to the specific clinical concern. The field-of-view must include the region of interest, with the foot usually divided into forefoot, midfoot or hindfoot regions. ${ }^{(19)}$ A small field-of-view and thin sections optimise spatial resolution, while a large field-of-view sacrifices detail and should be avoided. Nevertheless, the entire foot may be scanned to include the full extent of infection. The foot should be examined clinically prior to MR imaging. If possible, external markers should be positioned over shallow ulcers, which may otherwise be difficult to identify on the images (Fig. 1). Similarly, external markers should be placed over suspected sinus tract cutaneous openings. The foot is placed in an extremity coil and images should be acquired in at least two planes in order to adequately visualise the region of interest. Each plane has its advantages. For example, the sagittal plane is suitable for depicting midfoot neuroarthropathic changes and posterior calcaneal ulcers; the axial and coronal planes are useful for demonstrating medial and lateral hindfoot ulcers; and a plane aligned perpendicular to the toes (short-axis view) is best suited for imaging toe ulcers and their relationship with the adjacent bones.
T1-weighted (T1-W) sequences depict anatomical detail well. They are sensitive to marrow changes and are the most specific in the detection of osteomyelitis. In addition, T1-W images aid in the identification of blood products. T2-weighted (T2-W) sequences are exquisitely sensitive in the detection of oedema (both bone marrow and soft tissue) and fluid collections. However, with fast spin-echo technique, the bright signal of marrow oedema may be masked by the bright signal of fatty bone marrow, potentially causing diagnostic error. Acquiring T2-W sequences with chemical fat suppression solves this problem; however, to achieve homogeneous fat suppression, the foot has to be positioned in the centre of the magnet. Short tau inversion recovery (STIR) sequence is an alternative, as it gives more uniform fat suppression due to the curvature of the foot, and at the same time remains extremely sensitive to marrow and soft tissue oedema. However, the image resolution is usually less optimal on STIR sequences.

Intravenous gadolinium contrast medium can be given, but its routine use is debatable. Some studies recommend its routine use, ${ }^{(15,20)}$ while others suggest it is unnecessary. ${ }^{(7,21,22)}$ There is no conclusive evidence that intravenous contrast administration increases the accuracy of detection of osteomyelitis, although 
it certainly improves the evaluation of soft tissue pathology (e.g. abscesses, sinus tracts) and allows distinction between viable and nonviable soft tissue or bone. Hence, it is useful in identifying secondary signs of osteomyelitis and is of value in preoperative planning. In our centre, we routinely acquire post-contrast MR imaging, particularly if abnormal signal is present on routine T1- and T2-W MR imaging.

\section{SOFT TISSUE COMPLICATIONS}

\section{Skin callus}

Subcutaneous fat is redistributed from the sole of the diabetic foot, changing the biomechanics and leading to skin callus formation at weight-bearing sites. ${ }^{(12)}$ This is exacerbated by friction caused by ill-fitting footwear. In ambulatory patients, skin calluses tend to occur over the metatarsal heads (particularly the first and fifth metatarsals), the tip of the big toe, the distal toes, the tarsometatarsal joints, the cuboid (with the presence of 'rockerbottom' deformity), tendo-Achilles bursa and malleoli. ${ }^{(23,24)}$ In bed-bound patients, as the foot tends to be externally rotated and subjected to longstanding pressure effects, skin calluses have a propensity to occur over the calcaneum and lateral malleolus.

On MR images, skin calluses manifest as focal subcutaneous lesions, showing low T1-W signal intensity and low to intermediate T2-W signal intensity. Enhancement of callus may mimic soft tissue infection, but the characteristic location and paucity of surrounding soft tissue signal change aid in differentiating it from infection. Adventitial bursa formation may occur over the same sites as calluses, since they are both caused by chronic friction. An adventitial bursa is typically seen overlying a bony prominence, appearing as a thin fluid collection with no accompanying subcutaneous inflammatory fat stranding (Fig. 1).

\section{Ulcer}

Breakdown of a skin callus results in an ulcer. Hence, ulcers are typically preceded by skin calluses. Exceptions include midfoot ulcers in the neuropathic foot and dorsal ulcers in toes with flexion deformities. These may occur without prior callus formation. In diabetic patients, multiple factors contribute to the breakdown of calluses. Sensory neuropathy, the primary contributing factor, results in unperceived trauma, which eventually leads to skin breakdown. Autonomic neuropathy causes skin dryness, which predisposes to fissuring and ulceration. Less commonly, motor neuropathy, which causes atrophy of the anterior calf muscles (foot dorsiflexors) or the intrinsic muscles of the foot, leading to deformities such as hammer toes, prominent plantar metatarsal heads, foot drop and equinus, may be present. ${ }^{(25)}$ These result in pressure points, which give rise to callus and ulcer formation.

On MR imaging, a skin ulcer appears as a local disruption of the cutaneous line, with associated raised margins (secondary to pre-existing callus formation) and soft tissue defects. Acute ulcers may demonstrate high T2-W signal intensity and peripheral enhancement, suggestive of granulation tissue at the ulcer base (Fig. 1). Chronic ulcers may appear as an induration of intermediate to low T2-W signal intensity in the subcutaneous layer, reflecting healing by fibrosis. Any interruption of the skin barrier, including subtle superficial skin erosions, can serve as a portal for infection. Skin ulcers deeper than $2 \mathrm{~cm}$ are especially prone to osteomyelitis. ${ }^{(23,26)}$

\section{Cellulitis}

Cellulitis refers to an acute non-necrotising inflammatory process involving the skin and subcutaneous tissue but spares the deep fascia and muscles. Clinically, there is soft tissue swelling, localised pain and tenderness, erythema and warmth, features that can be confused with or masked by oedematous change secondary to acute neuropathy. ${ }^{(4)}$

On MR imaging, cellulitis appears as loss of the normal subcutaneous fat signal intensity on T1-W images, with associated increased T2-W signal intensity and diffuse enhancement with intravenous gadolinium. ${ }^{(16,26)}$ The margins are typically ill defined. Cellulitis and acute neuropathy may appear similar on MR imaging, with skin thickening and more prominent reticulation of fat. ${ }^{(27)}$ However, enhancement is characteristic of cellulitis and typically not present in oedema of neuropathic disease (Figs. 1 \& 2). ${ }^{(28)}$

\section{Abscess and sinus tract}

Abscess formation can occur with chronic infections, or may be seen early in aggressive infections. Between 10\% ${ }^{(16)}$ and 50\% ${ }^{(29)}$ of patients with pedal infection show MR imaging evidence of abscesses. An abscess manifests as a rim-enhancing collection of fluid signal intensity ${ }^{(28)}$ (Fig. 3) and typically occurs close to a site of skin breakdown or in adjacent fascial compartments. It may, however, be remote from skin ulceration in established infections where there is a larger extent of spread.

The majority of abscesses are small, and in the absence of contrast administration, may be easily masked by surrounding soft tissue oedema. ${ }^{(28)}$ Hence, in the setting of infection, contrast administration is essential to aid abscess identification and show the extent of soft tissue infection. Sometimes, despite contrast administration, the abscess may lie in such close proximity to the skin that its enhancing rim is not discernible.

Abscesses are frequently contiguous with sinus tracts that communicate with skin ulcers, tendon sheaths, bones or joints. On post-contrast MR images, sinus tracts show a 'tram-track' pattern of enhancement. ${ }^{(15,30)}$ Sinus tracts require imaging evaluation in all planes - a sinus tract with a tortuous course may be erroneously labelled as an abscess, since it may appear circular in cross-section in a single plane (Fig. 4).

\section{Gangrene}

Gangrene refers to soft tissue necrosis. In diabetic patients, microvasculopathy causes ischaemia, which may in turn lead to gangrene. Most cases of gangrene are diagnosed clinically. The purpose of contrast-enhanced MR imaging is usually to delineate areas of soft tissue necrosis, which aids surgical planning. ${ }^{(31)}$ Gangrene is classified as either dry or wet, based on the absence or presence of infection, respectively. In wet gangrene, soft tissue 

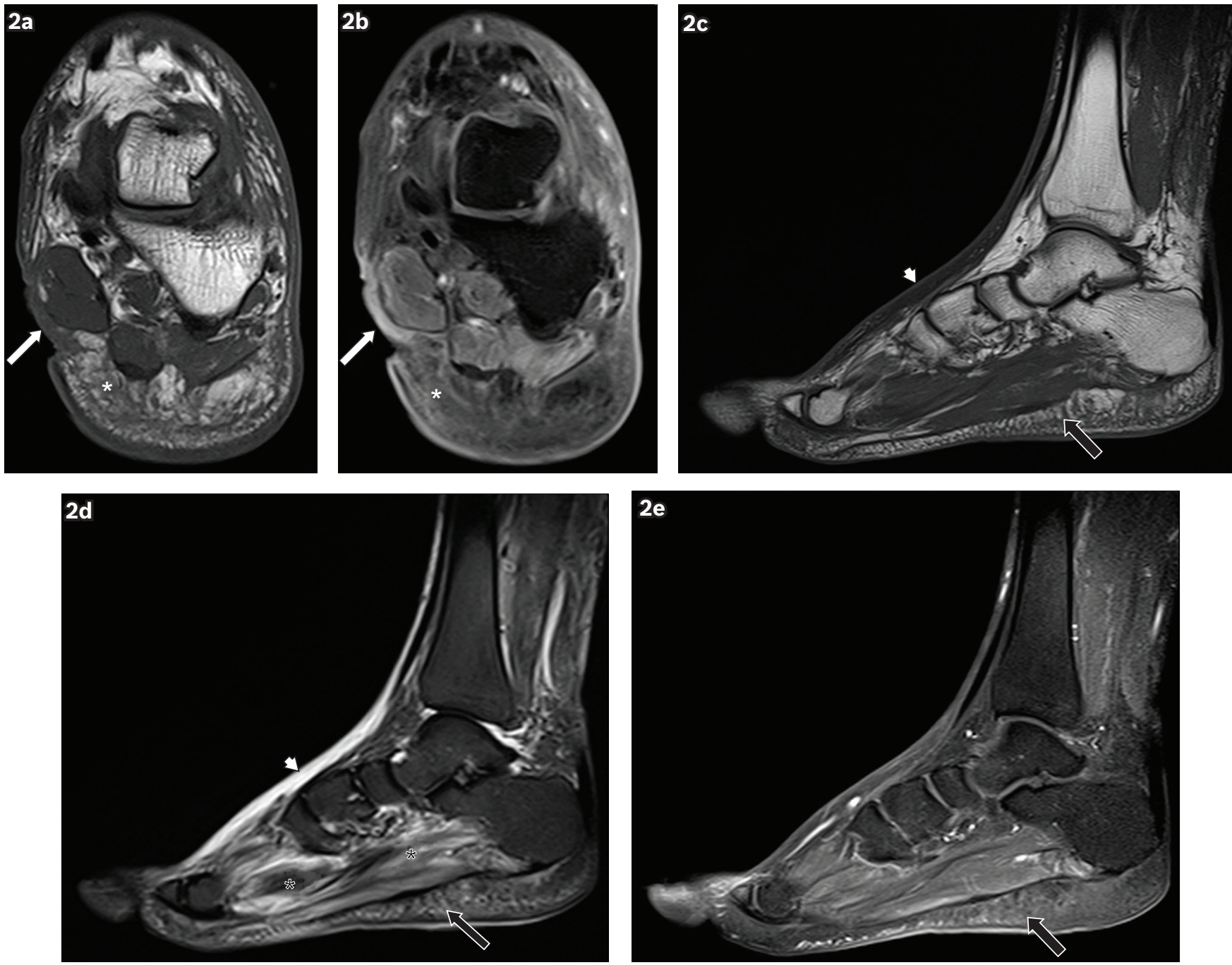

Fig. 2 A 62-year-old man presented with a wound on the medial aspect of the left hindfoot, sustained one month earlier, with pus discharge and surrounding inflammation. Coronal (a) T1-W and (b) contrast-enhanced T1-W FS MR images of the left hindfoot show an ulcer (white arrows) on the medial aspect of the hindfoot, seen as a soft tissue defect with focal interruption of the cutaneous line. Enhancing granulation tissue is noted at the base of the ulcer and surrounding cellulitis is present (white asterisks), seen as areas of T1-hypointense signal within the subcutaneous tissues, with prominent reticulation and enhancement of subcutaneous fat. Sagittal (c) T1-W, (d) T2-W FS and (e) contrast-enhanced T1-W FS MR images of the left foot show reactive subcutaneous oedema at the dorsum of the foot (arrowheads), which shows low T1-W and high T2-W signals, similar to those for cellulitis. However, no significant enhancement is seen, distinguishing it from cellulitis. Mild cellulitis is noted in the sole (black arrows), with T1 hypointensity, T2 hyperintensity and mild enhancement in the subcutaneous tissues. Increased T2-W signal in the plantar muscles is characteristic of myositis (black asterisks).

gas due to infection by gas-forming bacteria may be present. In such cases, there is usually rapid spread of infection and urgent surgical treatment is often necessary.

On post-contrast MR imaging, gangrene is seen as an area of unenhanced devitalised soft tissue that has a well-defined border with adjacent viable soft tissue. Reactive hyperaemia and enhancement may be present at the margins of the devitalised tissue. ${ }^{(31)}$ Necrotic tissue may liquefy, resulting in fluid signal intensity on MR imaging.

Gas associated with wet gangrene can be difficult to see on MR imaging, as it is found within fascial layers, which normally show low MR imaging signal intensity similar to gas. Gradientecho sequences have the highest sensitivity in the detection of soft tissue gas, which causes prominent blooming artefact (Fig. 5). The radiologist has to be careful in differentiating soft tissue gas secondary to wet gangrene from that associated with a skin ulcer. Wet gangrene typically shows unenhanced devitalised soft tissue, with a greater extent of soft tissue gas compared to that seen surrounding a skin ulcer. ${ }^{(32)}$

\section{Foreign body}

Patients with sensory neuropathy secondary to DM are predisposed to unperceived foreign body penetration of the foot. Foreign bodies are usually located under the metatarsal heads. They may also be seen after surgery. MR images should be scrutinised for a foreign body whenever there is soft tissue infection but no accompanying skin ulcer.

A foreign body is typically hypointense on both $\mathrm{T} 1$ - and T2-W sequences, and may show blooming artefact on gradientecho sequences. ${ }^{(19)}$ Granulomatous reaction results in peripheral enhancement, which should not be misinterpreted as an abscess. ${ }^{(33)} \mathrm{T} 2-\mathrm{W}$ sequences help to differentiate an abscess from a foreign body, since the former contains fluid (T2-hyperintense), while the latter does not. 

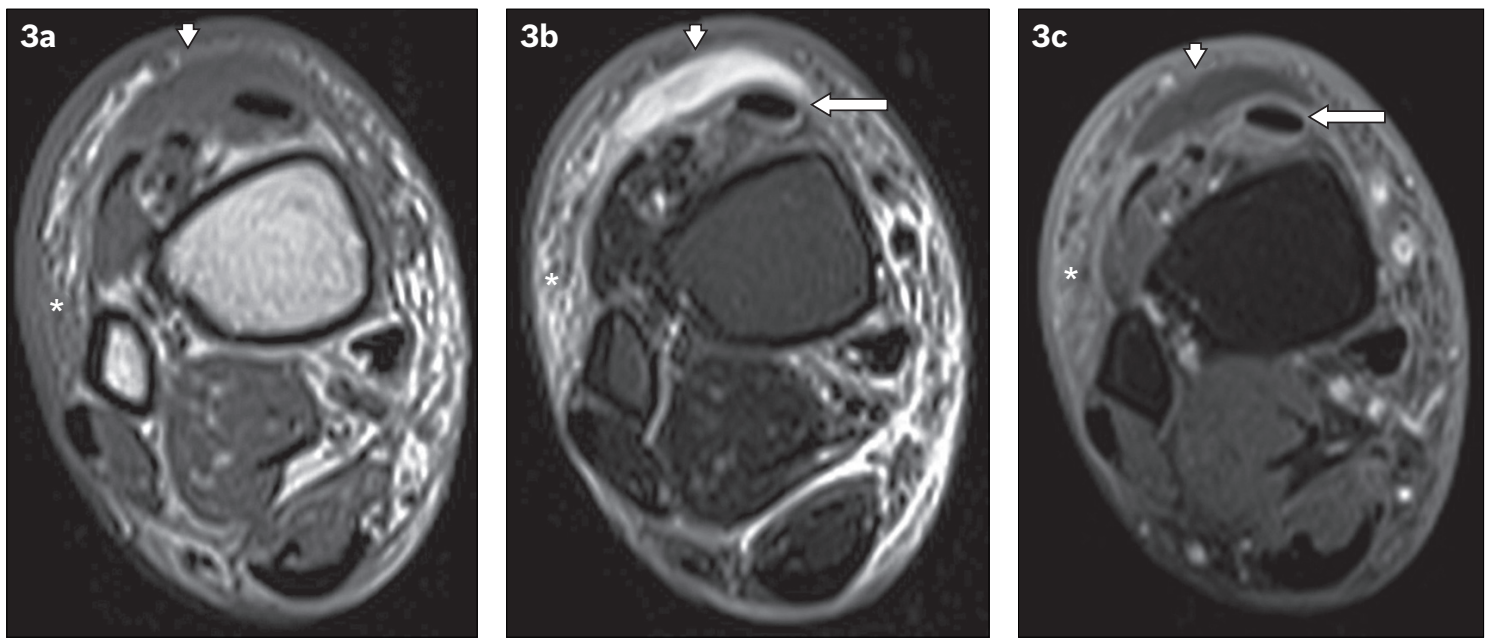

Fig. 3 A 60-year-old man presented with fever and painful swelling over the anterior aspect of the right ankle. Axial (a) T1-W, (b) T2-W FS and (c) contrastenhanced T1-W FS MR images of the right distal leg show a crescent-shaped T1-hypointense and T2-hyperintense fluid collection, with mild peripheral contrast enhancement anterior to the extensor tendons of the foot, consistent with a subcutaneous abscess (arrowheads). Surrounding cellulitis is observed, evident as areas of T1-hypointense and T2-hyperintense signals within the subcutaneous tissues with enhancement of subcutaneous fat (asterisks). Peritendinous high T2-W signal and enhancement involving the tibialis anterior and other extensor tendons is in keeping with tenosynovitis (arrows).
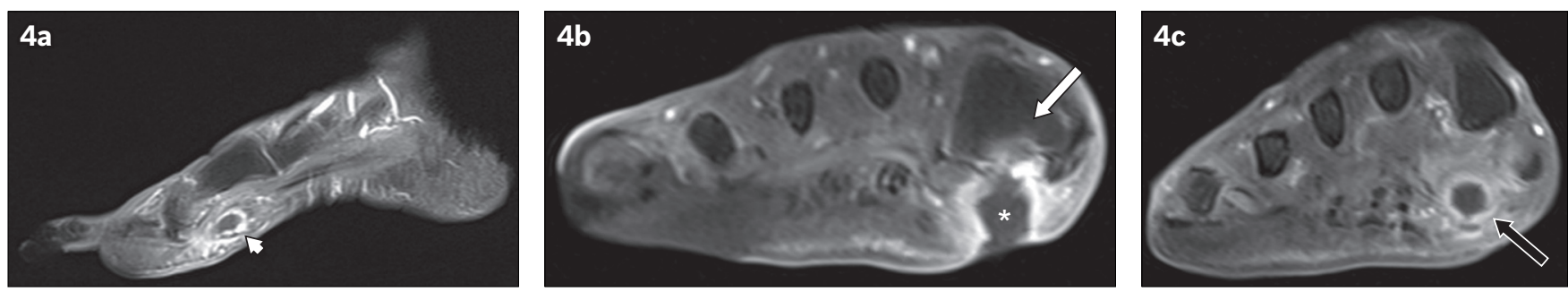

Fig. 4 A 79-year-old woman presented with fever and symptoms of right foot infection. (a) Sagittal contrast-enhanced T1-W FS MR image of the right foot shows the 'tram-track' pattern of enhancement of a sinus tract in the subcutaneous layer of the plantar forefoot (arrowhead). (b \& c) Coronal contrast-enhanced T1-W FS MR images of the right foot taken at two separate locations. (b) The sinus tract extends to a subcutaneous abscess (asterisk), adjacent to the base of the first metatarsal and with an overlying skin ulcer. The adjacent bone marrow enhances, suggesting osteomyelitis (white arrow). (c) Further distally in the right forefoot, the cross-sectional appearance of the sinus tract is demonstrated as a round rim-enhancing fluid collection, which may be mistaken for an abscess if not evaluated properly (black arrow).

\section{BONE/JOINT COMPLICATIONS Osteomyelitis}

At presentation, clinical findings of infection are seen in more than $50 \%$ of diabetic patients with skin ulcer of the foot. Depending on the severity, between $20 \%$ and $60 \%$ of these infections are accompanied by bone infection. ${ }^{(34-36)}$ The easiest way to detect osteomyelitis on MR imaging is to follow the path of the skin ulcer or sinus tract to the bone and assess the marrow signal intensity (Figs. 4 \& 6). Markedly low T1-W signal intensity is a key sign of osteomyelitis (Fig. 7). ${ }^{(15)}$ Secondary signs may confirm the diagnosis. These include the aforementioned soft tissue complications that suggest the presence of infection. Periosteal reaction is another secondary sign of osteomyelitis. On MR imaging, the low signal intensity calcified periosteum is lifted from the bone by a layer of fluid or pus. However, this finding is better appreciated on radiography, as compared to MR imaging.

Osteomyelitis should not be confused with osteitis, which refers to reactive marrow changes secondary to a neighbouring infection (of soft tissue or cortical bone). On T2-W images, osteitis appears as hyperintense marrow, which is identical to that seen in osteomyelitis. However, on T1-W images, it does not demonstrate the low signal that characterises osteomyelitis.
Bone marrow enhancement may be seen but is not reliable in discriminating between the two entities. ${ }^{(12)}$

MR imaging evaluation of the postsurgical diabetic foot is commonly performed, and the same principles apply when determining the presence of osteomyelitis at an amputation site. ${ }^{(19)}$ In the case of debridement, postsurgical marrow oedema may be present and should not be misinterpreted as osteomyelitis. The majority of amputations show minimal postsurgical marrow oedema.

\section{Neuroarthropathy}

Neuroarthropathy is an uncommon but severe complication of diabetic peripheral neuropathy, which is estimated to affect between $0.8 \%$ and $8.0 \%$ of the diabetic population. ${ }^{(37)} \mathrm{A}$ higher proportion of cases are found when advanced imaging studies are utilised for diagnosing foot problems, and the incidence of neuroarthropathy appears to be increasing. ${ }^{(37,38)}$ In the acute stage, neuroarthropathy is treated by immobilisation and pressure offloading, usually with concomitant pharmacotherapy (e.g. oral/ intravenous bisphosphonates, nasal calcitonin). In the complicated diabetic foot, it is often difficult to differentiate osteomyelitis and neuroarthropathy, especially at initial presentation. Since their management is significantly different and largely determines 

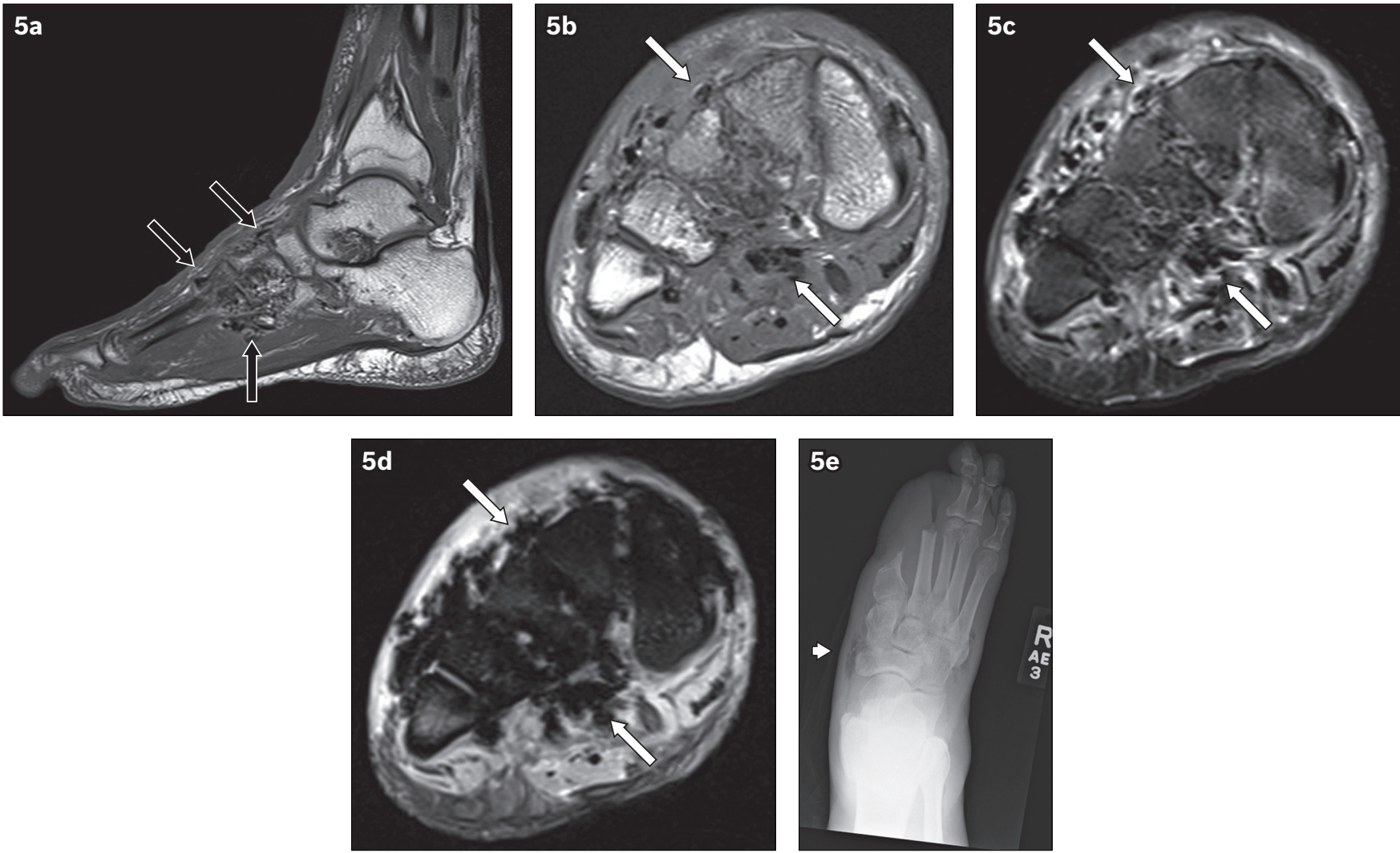

Fig. 5 A 62-year-old man presented with the clinical diagnosis of wet gangrene of the right foot. Signs of infection and crepitation were elicited on examination of the right foot. (a) Sagittal T1-W MR image of the right foot shows low signal foci of magnetic susceptibility along the fascial planes in the midfoot (black arrows), in keeping with soft tissue gas. Axial (b) T1-W, (c) T2-W FS and (d) gradient-echo MR images of the right midfoot show soft tissue gas, seen as low signal foci of magnetic susceptibility (white arrows). The gradient-echo sequence is very sensitive in the detection of gas - the magnetic susceptibility caused by gas manifests as blooming artefacts on this MR imaging sequence, making them more conspicuous. (e) Frontal radiograph of the right foot, obtained at the same time, reveals lucencies in the soft tissues of the midfoot (arrowhead), in keeping with gas and corresponding to the MR imaging appearances. The patient was status post partial amputation of first and second rays.
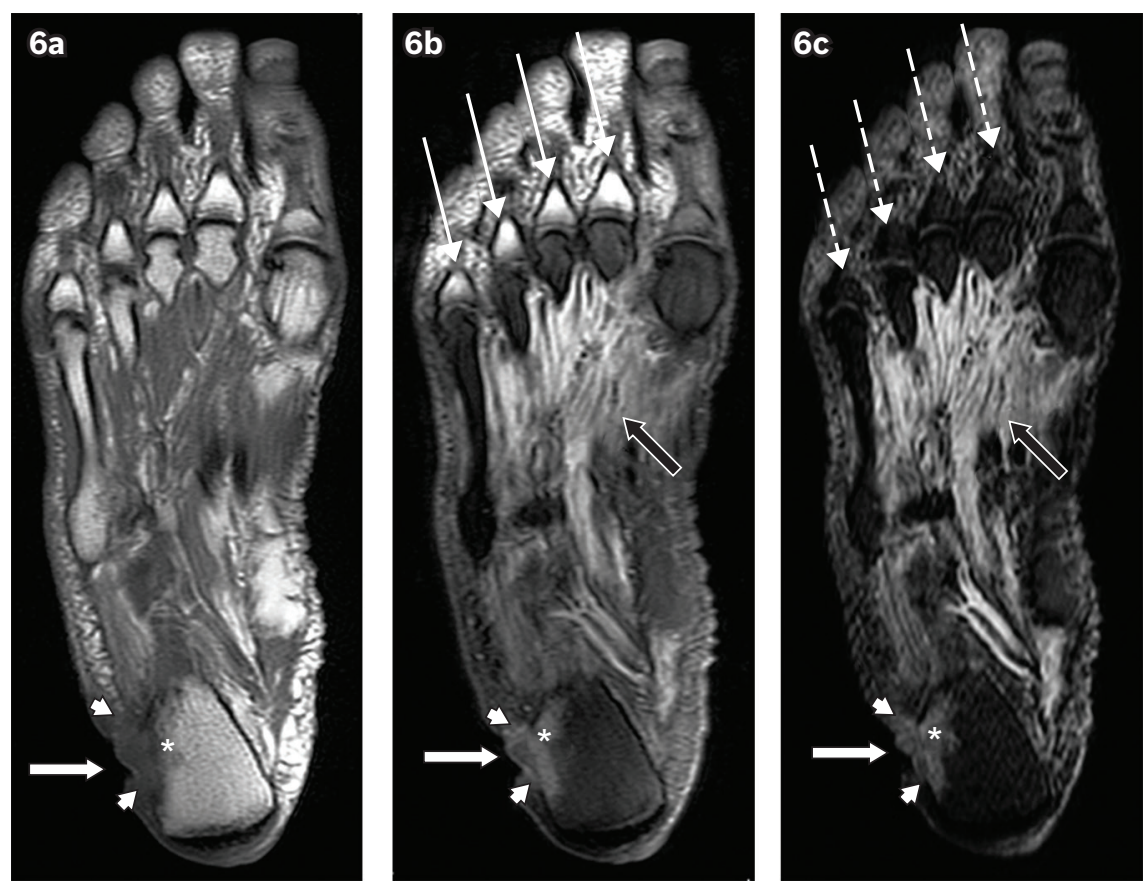

Fig. 6 A 60-year-old man presented with a chronic ulcer on the lateral aspect of the right heel, with symptoms of infection. Axial (a) T1-W, (b) T2-W FS and (c) STIR MR images of the right foot show an ulcer (white arrows) along the lateral aspect of the heel with surrounding cellulitis (arrowheads). Low T1-W and high T2-W marrow signals in the adjacent calcaneum, with associated cortical ill-definition, suggest osteomyelitis (asterisks). Notice also the reactive myositis involving the interosseous muscles, seen as high signal on T2-W FS and STIR sequences (black arrows). High T2-W signal in the visualised proximal phalanges on the T2-W FS image (b) is due to failure of fat suppression (thin arrows), a potential pitfall in MR imaging. The corresponding areas on the STIR sequence (c) show better fat suppression, with appropriate signal drop-out of normal marrow (broken arrows). 

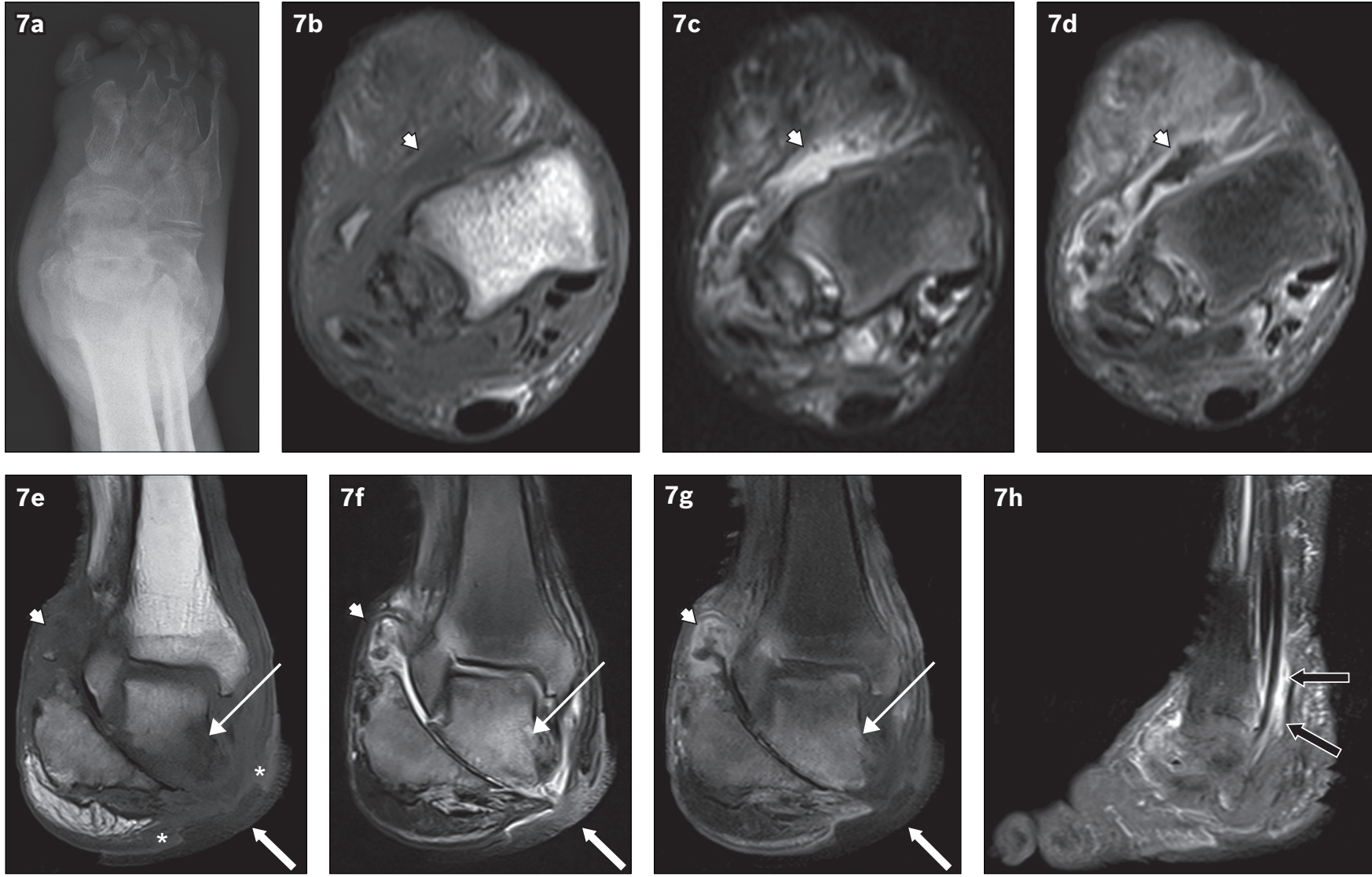

Fig. 7 An 81-year-old man presented with a chronic nonhealing right heel ulcer with foul-smelling discharge and surrounding inflammation. (a) Frontal radiograph of the right foot shows changes of chronic neuroarthropathy, with bony resorption of the proximal phalanges and metatarsals, and disorganisation of the subtalar and intertarsal joints. Axial (b) T1-W, (c) T2-W FS and (d) contrast-enhanced T1-W FS, and coronal (e) T1-W, (f) T2-W FS and (g) contrast-enhanced T1-W FS MR images of the right foot are shown. Chronic neuroarthropathy is most strikingly illustrated by disruption of the subtalar joint, seen on the coronal images. An ulcer is present on the medial aspect of the hindfoot (white arrows), with the disrupted subtalar joint deep to it. Fluid collections are seen lining the ankle and subtalar joints, showing high T2-W and low T1-W signals with associated synovial outpouchings and contrast enhancement, consistent with synovitis (arrowheads). In isolation, these imaging findings cannot distinguish septic arthritis or superimposed infection from pure neuroarthropathy. However, in this case, there is an area of marked low T1-W signal involving the inferior and medial portions of the talus with high signal on post-contrast and T2-W images (thin arrows), suggesting superimposed osteomyelitis and the presence of infection. The 'ghost sign' is present, with the involved bones more clearly delineated on the T2-W FS and contrast-enhanced T1-W FS images, compared to the T1-W sequence (in which the bones 'disappear'). In addition, the absence of adjacent subcutaneous fat signal intensity is suggestive of superimposed infection (asterisks). (h) Sagittal contrast-enhanced T1-W FS MR image of the right foot shows peritendinous contrast enhancement along the tibialis posterior, flexor digitorum longus and flexor hallucis longus tendons, suggestive of tenosynovitis (black arrows).

patient outcome, it is important for clinicians and radiologists to know how to diagnose these conditions.

It may be challenging to distinguish early neuroarthropathy from osteomyelitis on clinical examination, bone scintiscans and MR imaging. During the early phases of neuroarthropathy, when radiographs are normal, MR imaging may show soft tissue oedema, joint effusions, fluid collections and abnormal marrow signal intensity. ${ }^{(7,39)}$ Post-contrast MR imaging reveals enhancement of the marrow and periarticular soft tissue ${ }^{(40)}$ (Fig. 8). During the subacute stage of neuroarthropathy, bone resorption occurs. During the chronic stage, deformity and osseous fragmentation occur. On MR imaging, chronic neuroathropathy may show joint effusion with mild marrow oedema and no significant soft tissue oedema. On clinical examination, this chronic and inactive stage of neuroarthropathy does not resemble osteomyelitis, so any clinical feature of infection should raise the possibility of osteomyelitis.

\section{Differentiating osteomyelitis from neuroarthropathy}

While neuroarthropathy is primarily articular, osteomyelitis virtually always occurs by direct extension from skin ulcers at typical sites. The presence of marrow oedema and periarticular disease with no adjacent ulcer is highly suggestive of neuroarthropathy. Superimposed osteomyelitis usually shows diffuse marrow changes, whereas neuroarthropathy often shows periarticular marrow changes, since the pathology centres on the joint (Fig. 8). Location is the most helpful feature in discriminating between osteomyelitis and neuroarthropathy. Most of the time, neuroarthropathy involves the tarsometatarsal and metatarsophalangeal joints, while osteomyelitis commonly involves the calcaneum, malleoli and bones distal to the tarsometatarsal joint. The biggest diagnostic problem arises in the midfoot, particularly when pre-existing neuroarthropathy is present. In such cases, secondary signs of infection are invaluable in determining the presence of osteomyelitis.

\section{Neuroarthropathy with superimposed infection}

In the presence of skin ulceration extending to the bone, patients with pre-existing neuroarthropathy more commonly have osteomyelitis compared to patients without. Although the specificity of MR imaging in detecting osteomyelitis may 

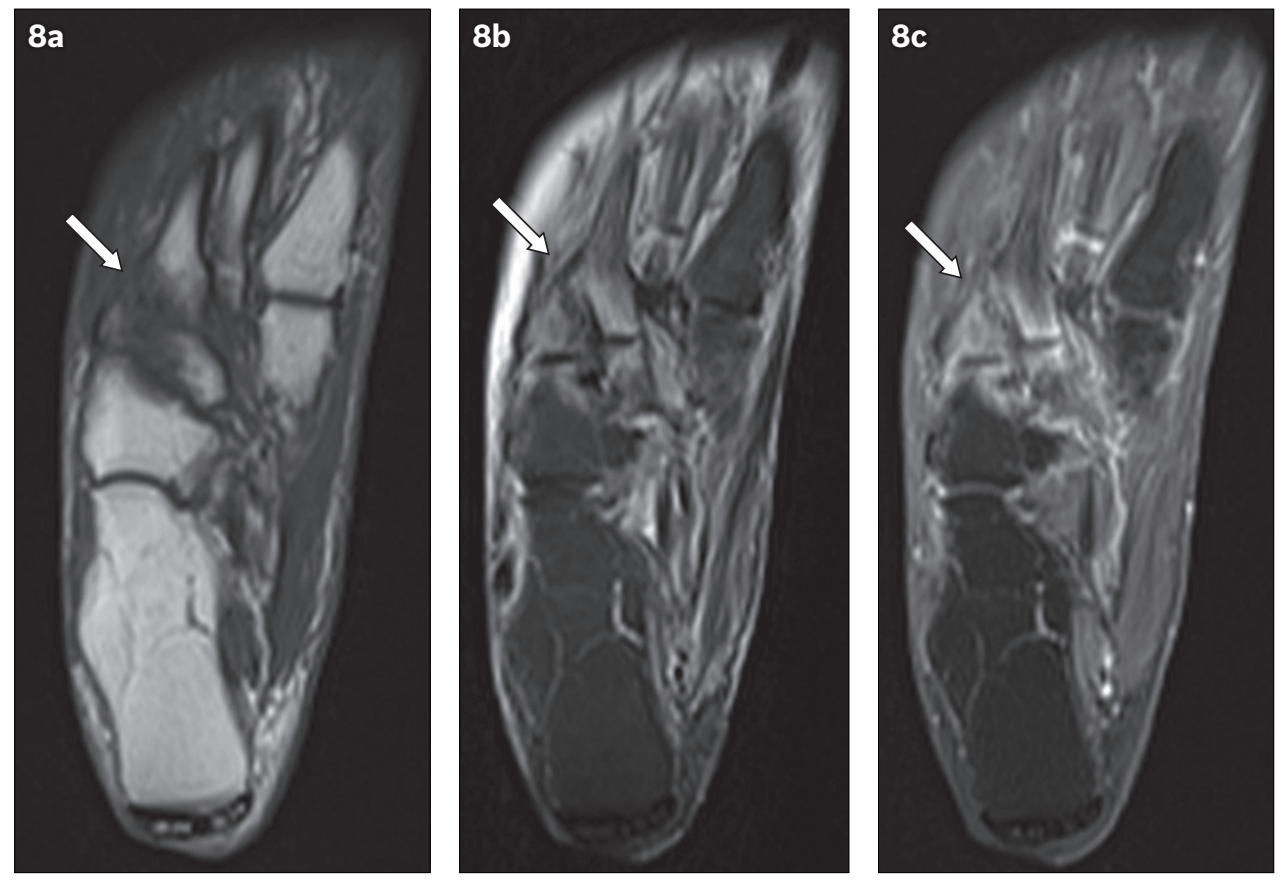

Fig. 8 An 81-year-old woman presented with tenderness over the dorsum of the right foot, with associated inflammatory skin changes. Axial (a) T1-W, (b) T2-W FS and (c) contrast-enhanced T1-W FS MR images of the right foot show periarticular high T2-W signal with corresponding enhancement, centred around the tarsometatarsal joints (arrows). The typical location, coupled with the absence of other secondary soft tissue signs of infection (e.g. ulcer, cellulitis, abscess, sinus tract), indicated the diagnosis of acute neuroarthropathy. The patient was given analgesia for symptomatic treatment and her symptoms subsequently resolved.

be limited by pre-existing neuroarthropathy, in such cases, MR imaging is mainly used to assess disease extent rather than establish the diagnosis. ${ }^{(41)}$ Post-contrast MR imaging is recommended for the evaluation of soft tissue disease extent. ${ }^{(10)}$

Several imaging features help differentiate neuroarthropathy with superimposed infection from uncomplicated neuroarthropathy. In superimposed joint infection, soft tissue features that may be seen include a loss of fat signal intensity in the adjacent subcutaneous tissue (Figs. $7 \&$ 9) and the presence of neighbouring fluid collections larger than that expected for uncomplicated neuroarthropathy. Sinus tracts also occur frequently in superimposed infection, causing fluid collections to become smaller on subsequent imaging. Features such as skin ulcers, enhancement of soft tissue and periarticular rim-enhancing fluid collections are not useful in distinguishing neuroarthropathy from superimposed infection ${ }^{(12)}$ (Fig. 7). The disappearance of intra-articular bodies or subchondral cysts on follow-up imaging of patients with neuroarthropathy indicates a superimposed infection. ${ }^{(8)}$ Correspondingly, the presence of these two features on follow-up imaging may help to exclude superimposed osteomyelitis.

The 'ghost sign' is another useful feature that distinguishes uncomplicated neuroarthropathy from infected neuroarthropathy. The presence of the ghost sign points toward superimposed infection, while its absence excludes superimposed infection. The ghost sign refers to bones that 'disappear' on T1-W sequences and 'reappear' (become better delineated) on T2-W or post-contrast sequences ${ }^{(12)}$ (Figs. 7 \& 9). Infected bone apparently disappear on T1-W images due to their low signal intensity, but are clearly visualised on $\mathrm{T} 2-\mathrm{W}$ or post- contrast images. In contrast, true destruction of bones occurs in uncomplicated neuroarthropathy, resulting in the absence of the ghost sign.

\section{Septic arthritis}

Septic arthritis in the diabetic foot is typically a result of direct spread from soft tissue infection. ${ }^{(23,42)}$ Septic arthritis from haematogenous spread is slightly more common in diabetic patients than in the nondiabetic population. Nevertheless, most cases of septic arthritis in the diabetic foot are due to direct contiguous spread. Frequently, involved joints lie adjacent to the typical sites of skin callus or ulceration, which includes the ankle, subtalar, metatarsophalangeal and interphalangeal joints, and midfoot joints in the presence of background neuroarthropathy. Midfoot septic arthritis presents the greatest diagnostic challenge, since its imaging appearance can be almost identical to that of midfoot neuroarthropathy (Fig. 7).

On MR imaging, complex joint effusion with marked synovial enhancement is seen. Thickening and outpouchings of the synovium frequently occur. Contiguous extension of joint fluid to an adjacent sinus tract may be seen; in such cases, due to decompression, the joint effusion may become smaller at subsequent imaging even with persisting septic arthritis. There may be perisynovial oedema of the surrounding soft tissue, as well as reactive subchondral marrow oedema with marginal erosion. ${ }^{(43)}$ It is crucial to differentiate between reactive marrow changes and superimposed osteomyelitis. The former usually shows a thin band of subchondral marrow oedema, while the latter more commonly demonstrates marrow oedema extending proximally beyond the subchondral bone with marked hypointense marrow signal on T1-W images. ${ }^{(44)}$ 

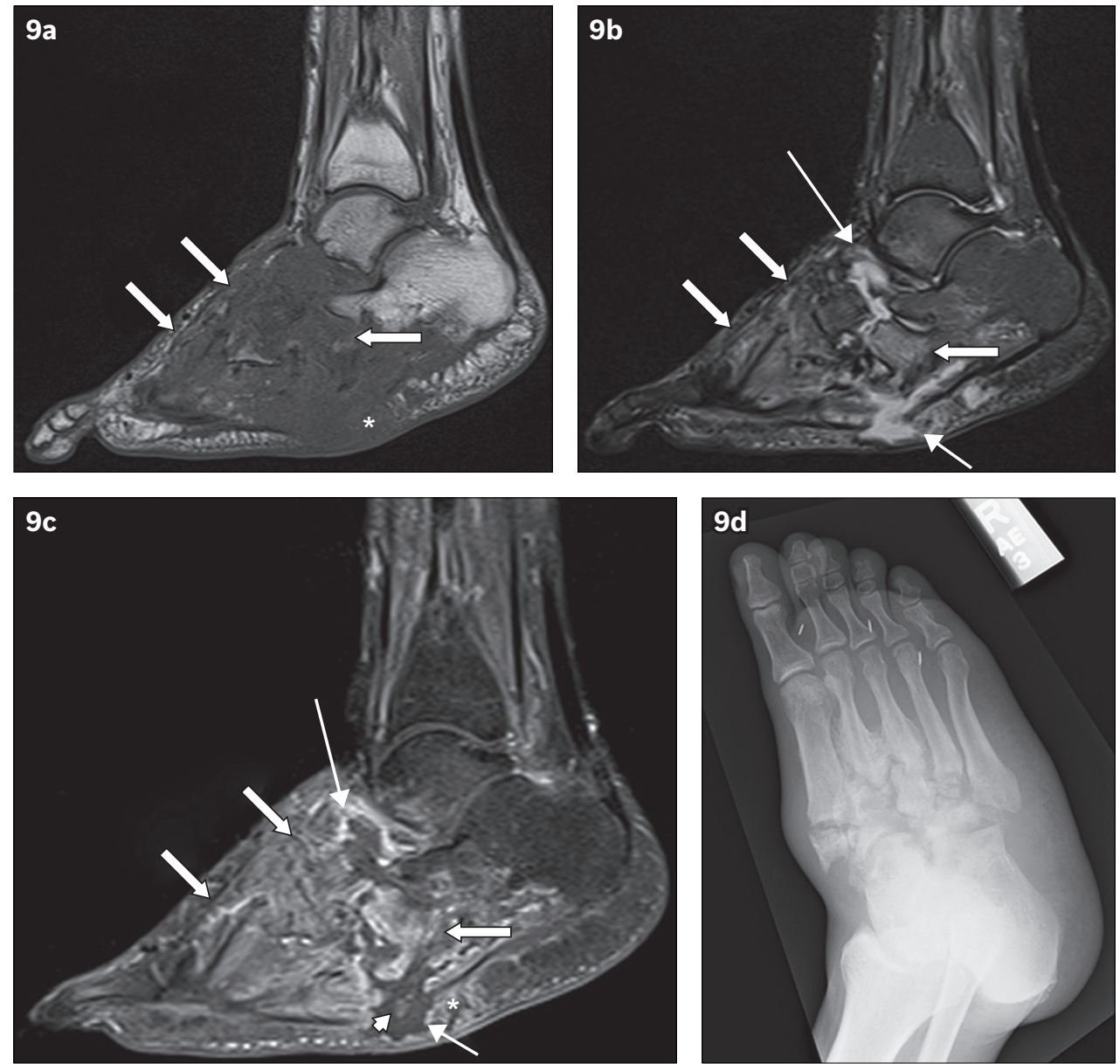

Fig. 9 A 49-year-old man presented with low-grade fever and signs of right foot infection. Sagittal (a) T1-W, (b) T2-W FS and (c) contrast-enhanced T1-W FS MR images of the right foot show extensive bony destruction centred around the midfoot joints, consistent with chronic neuroarthropathy. This is well demonstrated on the accompanying (d) oblique radiograph of the right foot. On the MR images, abnormal marrow signal is seen diffusely in the bones of the midfoot, extending beyond the subchondral bone; the affected bones show marked low T1-W signal and corresponding high T2-W signal as well as enhancement (arrows). The 'ghost sign' is present, with better delineation of the involved bones on the T2-W FS and contrast-enhanced T1-W FS images, compared to the T1-W image (in which the bones 'disappear'). MR imaging appearances suggest osteomyelitis. Secondary signs of infection are present, with a rim-enhancing abscess tracking into the intertarsal joints and extending to the subcutaneous layer via a sinus tract (thin arrows). Surrounding soft tissue enhancement and the absence of subcutaneous fat signal intensity are noted (asterisks). There is suggestion of a sequestrum (arrowhead).
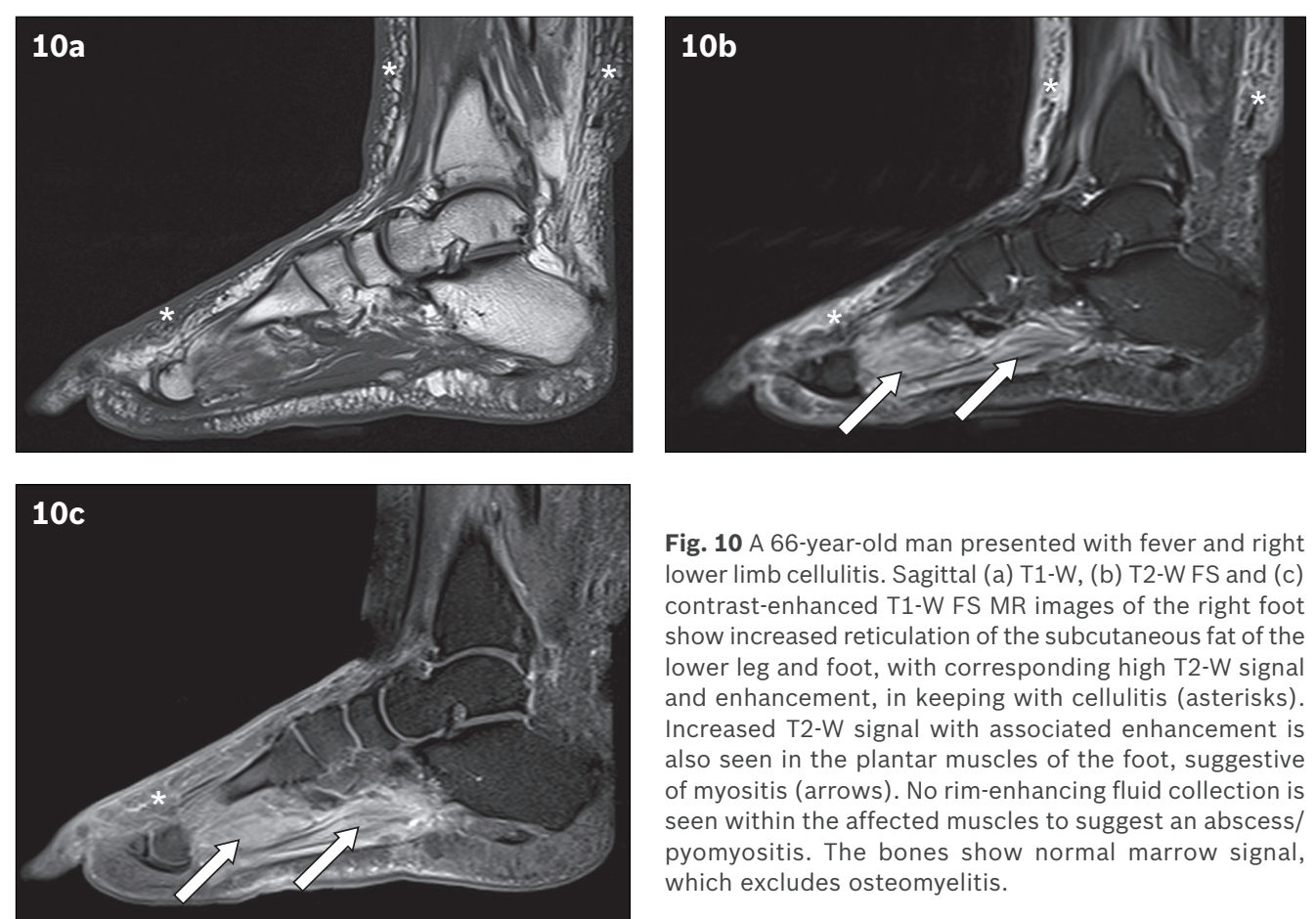

Fig. $10 \mathrm{~A}$ 66-year-old man presented with fever and righ lower limb cellulitis. Sagittal (a) T1-W, (b) T2-W FS and (c) contrast-enhanced T1-W FS MR images of the right foot show increased reticulation of the subcutaneous fat of the lower leg and foot, with corresponding high T2-W signal and enhancement, in keeping with cellulitis (asterisks). Increased T2-W signal with associated enhancement is also seen in the plantar muscles of the foot, suggestive of myositis (arrows). No rim-enhancing fluid collection is seen within the affected muscles to suggest an abscess/ pyomyositis. The bones show normal marrow signal, which excludes osteomyelitis. 


\section{MUSCULOTENDINOUS COMPLICATIONS} Tenosynovitis

Septic tenosynovitis typically occurs next to an ulcer or sinus tract due to local extension of infection. Most cases of septic tenosynovitis involves the peroneal tendons (adjacent to lateral malleolus skin ulceration) and the Achilles tendon (adjacent to calcaneal skin ulceration). ${ }^{(12)}$ In the forefoot, almost two out of three cases of tenosynovitis affect the flexor tendons, as a consequence of plantar skin ulcers.

On MR imaging, tenosynovitis is suggested by peritendinous enhancement within a region of cellulitis and associated with an infected skin ulcer ${ }^{(45)}$ (Figs. 3 \& 7). Thickening, T2-hyperintensity and enhancement of tendon are nonspecific findings - they may be secondary to infection but may also be present in other disease processes such as neoplastic, inflammatory or post-traumatic pathologies. ${ }^{(42)}$ Tenosynovitis is associated with osteomyelitis, likely because both entities are more commonly seen with advanced infection. ${ }^{(42)}$

A fluid-distended tendon sheath can also be seen in tenosynovitis, with the signal intensity of the fluid varying depending on its contents (e.g. the presence of debris, gas or blood). Fluid within a tendon sheath can be a normal finding, or may be seen in noninfective pathologies such as traumatic and mechanical conditions. However, fluid within the anterior tendon sheaths is considered neither normal nor caused by a mechanical condition. ${ }^{(12)}$

\section{Denervated muscle}

Patients with DM are predisposed to peripheral neuropathies, which can eventually lead to denervation of muscle. In the acute phase, muscle denervation may not show any abnormality on MR imaging. In the subacute phase, diffuse oedema is seen in the affected muscle, which appears hyperintense on T2-W and inversion recovery sequences. This finding is typically not seen on MR imaging for up to about two to four weeks after the onset of muscle denervation. ${ }^{(46)}$

Timely restoration of muscle innervation may lead to reversal of changes. Otherwise, irreversible chronic atrophy with associated infiltration of fat may result. ${ }^{(26)}$ On MR imaging, a larger amount of fat (with typical high T1-W signal intensity) is seen within the involved muscle bellies, with corresponding reduced muscle bulk. In the evaluation of chronic muscle denervation, T1-W sequences are more dependable, as variable appearances can be seen on T2-W and inversion recovery sequences. ${ }^{(46)}$

\section{Bacterial myositis/pyomyositis}

Myositis may be reactive (inflammatory) or bacterial (infective), and is frequently seen in association with other signs of soft tissue infection (Figs. 2, 6 \& 10). It is evident as oedema (high T2-W signal), with or without enhancement of the involved muscle. Bacterial myositis may show oedema as the only MR imaging abnormality, and may not be distinguishable from reactive myositis.

Bacterial myositis may be secondary to contiguous spread of infection from adjacent tissues (e.g. subcutaneous abscess and osteomyelitis). It commonly leads to intramuscular abscess formation, i.e. pyomyositis. ${ }^{(47)}$ In pyomyositis, focal rim-enhancing lesions can be seen on a background of diffuse high T2-W signal (oedema), with associated irregular enhancement of the deep fascia. ${ }^{(48)}$

\section{CONCLUSION}

MR imaging is increasingly available and currently the imaging modality of choice in the assessment of osteomyelitis and soft tissue complications in the diabetic foot. Due to the myriad manifestations and at times overlapping features of these complications, diagnosis via MR imaging can be challenging. It was the objective of this article to illustrate the MR imaging appearances and provide a strategy in the interpretation of MR images of diabetic foot complications. Familiarity with the specific technical requirements of MR imaging and the imaging features of these conditions will enable the radiologist to contribute significantly to patient management.

\section{REFERENCES}

1. Lam DW, LeRoith D. The worldwide diabetes epidemic. Curr Opin Endocrinol Diabetes Obes 2012; 19:93-6.

2. Lipsky BA, Pecoraro RE, Wheat LJ. The diabetic foot. Soft tissue and bone infection. Infect Dis Clin North Am 1990; 4:409-32.

3. Stadelmann WK, Digenis AG, Tobin GR. Impediments to wound healing. Am J Surg 1998; 176(2A suppl):39S-47S.

4. Rathur HM, Boulton AJ. The neuropathic diabetic foot. Nat Clin Pract Endocrinol Metab 2007; 3:14-25.

5. Chen SC, Huang SC, Wu CT. Nonspinal tuberculous osteomyelitis in children. J Formos Med Assoc 1998; 97:26-31.

6. Güttler A, Hammerschmidt S, Wirtz H, Schauer ]. [Unexpected cause of a tarsal destruction in a diabetic patient]. Dtsch Med Wochenschr 2004; 129:1243-5. German.

7. Marcus CD, Ladam-Marcus VJ, Leone J, et al. MR imaging of osteomyelitis and neuropathic osteoarthropathy in the feet of diabetics. Radiographics 1996; 16:1337-48.

8. Tan PL, Teh J. MRI of the diabetic foot: differentiation of infection from neuropathic change. Br J Radiol 2007; 80:939-48.

9. Tomas MB, Patel M, Marwin SE, Palestro CJ. The diabetic foot. Br J Radiol 2000; 73:443-50.

10. Schweitzer ME, Daffner RH, Weissman BN, et al. ACR Appropriateness Criteria on suspected osteomyelitis in patients with diabetes mellitus. J Am Coll Radiol 2008; 5:881-6.

11. Kapoor A, Page S, Lavalley M, Gale DR, Felson DT. Magnetic resonance imaging for diagnosing foot osteomyelitis: a meta-analysis. Arch Intern Med 2007; 167:125-32.

12. Donovan A, Schweitzer ME. Use of MR imaging in diagnosing diabetesrelated pedal osteomyelitis. Radiographics 2010; 30:723-36.

13. Levine SE, Neagle CE, Esterhai JL, Wright DG, Dalinka MK. Magnetic resonance imaging for the diagnosis of osteomyelitis in the diabetic patient with a foot ulcer. Foot Ankle Int 1994; 15:151-6.

14. Croll SD, Nicholas GG, Osborne MA, Wasser TE, Jones S. Role of magnetic resonance imaging in the diagnosis of osteomyelitis in diabetic foot infections. J Vasc Surg 1996; 24:266-70.

15. Morrison WB, Schweitzer ME, Bock GW, et al. Diagnosis of osteomyelitis: utility of fat-suppressed contrast-enhanced MR imaging. Radiology 1993; 189:251-7.

16. Unger E, Moldofsky P, Gatenby R, Hartz W, Broder G. Diagnosis of osteomyelitis by MR imaging. AJR Am J Roentgenol 1988; 150:605-10.

17. Berendt AR, Lipsky B. Is this bone infected or not? Differentiating neuroosteoarthropathy from osteomyelitis in the diabetic foot. Curr Diab Rep 2004; 4:424-9.

18. Ranachowska C, Lass P, Korzon-Burakowska A, Dobosz M. Diagnostic imaging of the diabetic foot. Nucl Med Rev Cent East Eur 2010; 13:18-22.

19. Chatha DS, Cunningham PM, Schweitzer ME. MR imaging of the diabetic foot: diagnostic challenges. Radiol Clin North Am 2005; 43:747-59, ix.

20. Dangman BC, Hoffer FA, Rand FF, O'Rourke EJ. Osteomyelitis in children: gadolinium-enhanced MR imaging. Radiology 1992; 182:743-7. 
21. Craig JG, Amin MB, Wu K, et al. Osteomyelitis of the diabetic foot: MR imaging-pathologic correlation. Radiology 1997; 203:849-55.

22. Miller TT, Randolph DA Jr, Staron RB, Feldwan F, Cushin S. Fat-suppressed MRI of musculoskeletal infection: fast T2-weighted techniques versus gadolinium-enhanced T1-weighted images. Skeletal Radiol 1997; 26:654-8.

23. Ledermann HP, Morrison WB, Schweitzer ME. MR image analysis of peda osteomyelitis: distribution, patterns of spread, and frequency of associated ulceration and septic arthritis. Radiology 2002; 223:747-55.

24. Mueller MJ, Minor SD, Diamond JE, Blair VP 3rd. Relationship of foo deformity to ulcer location in patients with diabetes mellitus. Phys Ther 1990; 70:356-62.

25. Frykberg RG. Diabetic foot ulcers: pathogenesis and management. Am Fam Physician 2002; 66:1655-62.

26. Tang JS, Gold RH, Bassett LW, Seeger LL. Musculoskeletal infection of the extremities: evaluation with MR imaging. Radiology 1988; 166:205-9.

27. Moore TE, Yuh WT, Kathol MH, el-Khoury GY, Corson JD. Abnormalities of the foot in patients with diabetes mellitus: findings on MR imaging. AJR Am J Roentgenol 1991; 157:813-6.

28. Ledermann HP, Morrison WB, Schweitzer ME. Pedal abscesses in patients suspected of having pedal osteomyelitis: analysis with MR imaging. Radiology 2002; 224:649-55.

29. Beltran J, Campanini DS, Knight C, McCalla M. The diabetic foot: magnetic resonance imaging evaluation. Skeletal Radiol 1990; 19:37-41.

30. Morrison WB, Schweitzer ME, Batte WG, Radack DP, Russel KM Osteomyelitis of the foot: relative importance of primary and secondary MR imaging signs. Radiology 1998; 207:625-32.

31. Ledermann HP, Schweitzer ME, Morrison WB. Nonenhancing tissue on MR imaging of pedal infection: characterization of necrotic tissue and associated limitations for diagnosis of osteomyelitis and abscess. AJR Am J Roentgenol 2002; 178:215-22.

32. Panchbhavi VK, Hecox SE. All that is gas is not gas gangrene: mechanica spread of gas in the soft tissues. A case report. J Bone Joint Surg Am 2006 88:1345-8.

33. Schweitzer ME, Morrison WB. MR imaging of the diabetic foot. Radio Clin North Am 2004; 42:61-71, vi.

34. Lavery LA, Peters EJ, Armstrong DG, et al. Risk factors for developing osteomyelitis in patients with diabetic foot wounds. Diabetes Res Clin Pract 2009; 83:347-52.

35. Lipsky BA, Peters EJ, Senneville E, et al. Expert opinion on the management of infections in the diabetic foot. Diabetes Metab Res Rev 2012; 28 Suppl 1:163-78.

36. Richard JL, Lavigne JP, Got I, et al. Management of patients hospitalized for diabetic foot infection: results of the French OPIDIA study. Diabetes Metab 2011; 37:208-15

37. Rajbhandari SM, Jenkins RC, Davies C, Tesfaye S. Charcot neuroarthropathy in diabetes mellitus. Diabetologia 2002; 45:1085-96.

38. Gouveri E, Papanas N. Charcot osteoarthropathy in diabetes: A brief review with an emphasis on clinical practice. World J Diabetes 2011; 2:59-65.

39. Morrison WB, Ledermann HP. Work-up of the diabetic foot. Radiol Clin North Am 2002; 40:1171-92.

40. Yuh WT, Corson JD, Baraniewski HM, et al. Osteomyelitis of the foot in diabetic patients: evaluation with plain film, 99mTc-MDP bone scintigraphy, and MR imaging. AJR Am J Roentgenol 1989; 152: 795-800.

41. Ledermann HP, Morrison WB, Schweitzer ME, Raikin SM. Tendon involvement in pedal infection: MR analysis of frequency, distribution, and spread of infection. AJR Am J Roentgenol 2002; 179:939-47.

42. Brower AC. Septic arthritis. Radiol Clin North Am 1996; 34:293-309, x.

43. Karchevsky M, Schweitzer ME, Morrison WB, Parellada JA. MRI findings of septic arthritis and associated osteomyelitis in adults. AJR Am J Roentgenol $2004 ; 182: 119-22$

44. Graif M, Schweitzer ME, Deely D, Matteucci T. The septic versus nonseptic inflamed joint: MRI characteristics. Skeletal Radiol 1999; 28:616-20.

45. Boutin RD, Brossmann J, Sartoris DJ, Reilly D, Resnick D. Update on imaging of orthopedic infections. Orthop Clin North Am 1998; 29:41-66.

46. Fleckenstein JL, Watumull D, Conner KE, et al. Denervated human skeletal muscle: MR imaging evaluation. Radiology 1993; 187:213-8.

47. Resnick D, Niwayama G. Osteomyelitis, septic arthritis, and soft tissue infection: mechanisms and situations. In: Resnick D, eds. Diagnosis of bone and joint disorders. 3rd ed. Philadelphia, PA: Saunders, 1995: 2325-418.

48. Chou H, Teo HE, Dubey N, Peh WC. Tropical pyomyositis and necrotizing fasciitis. Semin Musculoskelet Radiol 2011; 15:489-505. 


\section{SINGAPORE MEDICAL COUNCIL CATEGORY 3B CME PROGRAMME} (Code SMJ 201501B)

Question 1. Concerning the diabetic foot:

(a) Diabetic foot infections are rarely due to direct spread from a skin ulcer.

(b) The diabetic foot is prone to skin ulceration due to multiple risk factors.

(c) Diabetic patients are predisposed to progressive arthropathy (neuroarthropathy) of the foot.

(d) Infection of the soft tissue and bones is particularly common in the diabetic foot, and early diagnosis is crucial as it permits timely treatment.

Question 2. Regarding imaging of pedal osteomyelitis:

(a) Radiography is the preferred initial imaging modality.

(b) Magnetic resonance (MR) imaging is not useful, as it has poor sensitivity and specificity.

(c) Bone scintigraphy and white cell scintiscans have very high specificity.

(d) Ultrasonography has a limited role.

Question 3. Regarding soft tissue complications of the diabetic foot:

(a) In ambulatory patients, skin calluses tend to occur over the calcaneum rather than the metatarsal heads.

(b) Ulcers are rarely preceded by skin calluses.

(c) An abscess manifests as a rim-enhancing collection of fluid signal intensity.

(d) On contrast-enhanced MR imaging, gangrene is seen as an area of homogeneously enhancing soft tissue.

Question 4. Regarding bone and joint complications of the diabetic foot:

(a) Markedly low T1-weighted signal intensity is a key sign of osteomyelitis.

(b) Most cases of septic arthritis in the diabetic foot are due to haematogeneous spread.

(c) Periarticular marrow oedema, with no adjacent ulcer or secondary soft tissue signs of infection, is highly suggestive of neuroarthropathy.

(d) Osteomyelitis usually shows periarticular marrow changes, whereas neuroarthropathy shows diffuse marrow changes.

Question 5. Regarding musculotendinous complications of the diabetic foot:

(a) On MR imaging, tenosynovitis is suggested by enhancement of the tendon.

(b) Acute muscle denervation may not show any abnormality on MR imaging.

(c) Bacterial myositis may show oedema as the only MR imaging abnormality, and is easily distinguishable from reactive myositis.

(d) In pyomyositis, intramuscular abscess formation may be seen.

True

False

$\square$

\footnotetext{
Doctor's particulars:

Name in full

MCR number

Specialty:

Email address

\section{SUBMISSION INSTRUCTIONS:}

(1) Log on at the SMJ website: http://www.sma.org.sg/publications/smjcurrentissue.aspx and select the appropriate set of questions. (2) Provide your name, email address and MCR number. (3) Select your answers and click "Submit".

RESULTS:

(1) Answers will be published in the SMJ March 2015 issue. (2) The MCR numbers of successful candidates will be posted online at the SMJ website by 6 March 2015. (3) Passing mark is $60 \%$. No mark will be deducted for incorrect answers. (4) The SMJ editorial office will submit the list of successful candidates to the Singapore Medical Council. (5) One CME point is awarded for successful candidates.

Deadline for submission: (January 2015 SMJ 3B CME programme): 12 noon, 27 February 2015.
} 